\title{
An intronic variant in BRAT1 creates a cryptic splice site, causing epileptic encephalopathy without prominent rigidity
}

\author{
Fatma Kurt Colak $^{1} \cdot$ Naz Guleray $^{1} \cdot$ Ebru Azapagasi $^{2} \cdot$ Mutlu Uysal YazıcI $^{2} \cdot$ Erhan Aksoy $^{3} \cdot$ Nesrin Ceylan $^{3}$
}

Received: 18 July 2020 / Accepted: 28 September 2020 / Published online: 10 October 2020

(c) Belgian Neurological Society 2020

\begin{abstract}
BRAT1-related neurodevelopmental disorders are characterized by heterogeneous phenotypes with varying levels of clinical severity. Since the discovery of BRATl variants as the molecular etiology of lethal neonatal rigidity and multifocal seizure syndrome (RMFSL, OMIM 614498), these variants have also been identified in patients with milder clinical forms including neurodevelopmental disorder with cerebellar atrophy and with or without seizures (NEDCAS, OMIM 618056), epilepsy of infancy with migrating focal seizures (EIMFS), and congenital ataxia (CA). This study aims to examine the consequences and pathogenicity of a novel homozygous splice site variant in BRATl in a patient presenting with migrating focal seizures since birth without prominent rigidity. The patient was born from a consanguineous marriage and has had seizures since the neonatal period. He presented with dysmorphic features, pontocerebellar hypoplasia, and migrating focal seizures. Despite supportive treatment, his symptoms rapidly progressed to intractable myoclonic seizures, bouts of apnea and bradycardia, and arrest of head growth, with no acquisition of developmental milestones. Clinical exome sequencing yielded a novel homozygous splice variant in BRATI. Genetic analysis based on reverse transcription of the patient's RNA followed by PCR amplifications performed on synthesized cDNA and Sanger sequencing was undertaken, and the functional effect of a $B R A T 1$ variant on splicing machinery was demonstrated for the first time. The severe clinical presentation of migrating focal seizures and pontocerebellar hypoplasia in the absence of rigidity further expands the genotypic and phenotypic spectrum of BRAT1-related neurodevelopmental disorders.
\end{abstract}

Keywords BRAT1 Migrating focal seizure $\cdot$ Splice variant Pontocerebellar hypoplasia

\section{Introduction}

Epileptic encephalopathies are a genetically heterogeneous group of disorders characterized by continuous epileptiform

Electronic supplementary material The online version of this article (https://doi.org/10.1007/s13760-020-01513-0) contains supplementary material, which is available to authorized users.

Fatma Kurt Colak

drfatmakurt@gmail.com

1 Department of Medical Genetics, Dr. Sami Ulus Maternity and Children's Training and Research Hospital, Ankara, Turkey

2 Division of Pediatric Intensive Care Unit, Dr. Sami Ulus Maternity and Children's Training and Research Hospital, Ankara, Turkey

3 Department of Pediatric Neurology, Dr. Sami Ulus Maternity and Children's Training and Research Hospital, Ankara, Turkey activity on electroencephalogram (EEG) and by severe developmental delay. These encephalopathies are classified into different subgroups according to the age of onset, seizure types, and distinct EEG patterns. Over the past decade, broad application of next-generation sequencing has substantially accelerated the identification of diseaserelated genes as specific genetic encephalopathies have been defined with respect to characteristic electroclinical features and comorbidities [1]. Lethal neonatal rigidity and multifocal seizure syndrome (RMFSL, OMIM 614498) is a severe autosomal-recessive epileptic encephalopathy characterized by the onset of rigidity and intractable seizures at birth or soon after birth. This syndrome is caused by biallelic loss of function variants of BRAT1, which encodes BRCA1-associated ataxia telangiectasia mutated (ATM) activator 1 protein [2]. Neonates present with rigidity, microcephaly, myoclonic jerks, and multifocal refractory seizures leading to early death due to apnea and bradycardia [2-6]. RMFSL was first described in 2012; later, BRAT1 variants were also reported in patients with non-lethal milder clinical forms, including 
neurodevelopmental disorder with cerebellar atrophy and with or without seizures (NEDCAS, 618056), epilepsy of infancy with migrating focal seizures (EIMFS), and congenital ataxia (CA) [7-10]. We here report on a patient with a novel homozygous splice site variant in BRATl who on the first day of life presented with migrating focal seizures without prominent rigidity.

\section{Materials and methods}

\section{Clinical exome sequencing}

The protocol of this study was approved by the Kecioren Teaching and Research Hospital Clinical Research Ethics Committee (ID 2030). This study was conducted after obtaining written informed consent from the patient's parents. Genomic DNA (gDNA) was extracted from peripheral blood leukocytes of the proband and his healthy parents using a QIAamp DNA Mini Kit (Qiagen, Hilden, Germany). Furthermore, the Illumina TruSight One panel was used for clinical exome sequencing (CES) (Illumina, San Diego, CA, USA) and variant filtering steps were performed using SOPHiA ${ }^{\text {TM }}$ DDM (SOPHiA Genetics). Due to consanguinity, homozygous rare variants with low minor allele frequency values were prioritized. Additionally, a phenotype-driven bioinformatic analysis was performed. The pathogenicity of variants was assessed using ACMG criteria. Mutation Taster https://www.mutationtaster.org/ and Human Splicing Finder databases were used for predicting the pathogenicity of the splice site alterations.

\section{Reverse transcription reaction}

Total RNA of the proband and his healthy parents' samples were extracted from whole blood using the QIAamp RNA Blood Mini Kit (Qiagen, Hilden, Germany). Complementary DNA (cDNA) was reverse-transcribed (RT) from total RNA using ipsogen RT Kit (Qiagen, Hilden, Germany), following the manufacturer's protocol and recommended conditions.

\section{Polymerase chain reactions and Sanger sequencing}

Polymerase chain reactions (PCR) of cDNA sequences flanking splice site variant of $B R A T 1$ were performed using specific primers designed using the Perl Primer program. cDNA was PCR-amplified using a primer pair designed for the junction of exons 10 and 11 (forward primer), and for the junction of exons 13 and 14 (reverse primer) (Online Resource 1). To determine the pathogenicity of detected variant, PCR products of the cDNA samples were sequenced with BigDye ${ }^{\circledR}$ Terminator v3.1 Cycle Sequencing Kit
(Thermo Fisher Scientific, USA) on an ABI 3500 Genetic Analyzer (Thermo Fisher Scientific, USA). The sequences were aligned with the reference sequence of the BRATI (NCBI: NM_152743.4) gene.gDNA samples were used for the segregation analysis.

\section{Results}

\section{Case description}

The patient was born from the 5th pregnancy of a 25-yearold mother at full term after an uneventful pregnancy. Birth weight was $2350 \mathrm{~g}(-1.94 \mathrm{SD})$. The parents were healthy first-degree cousins of Turkish origin (Fig. 1A). Soon after birth, he was intubated due to respiratory failure and he was transferred to the neonatal intensive care unit (NICU). He developed his first seizure within $24 \mathrm{~h}$ of life. Neurometabolic disorder was suspected due to the coexistence of hypotonia and epilepsy. He was transferred to the pediatric intensive care unit (PICU) at the age of 45 days with priorly commenced antiepileptic treatment with vigabatrin (dosage: $120 \mathrm{mg} / \mathrm{kg} /$ day), levetiracetam (dosage: $60 \mathrm{mg} / \mathrm{kg} /$ day), topiramate (dosage: $5 \mathrm{mg} / \mathrm{kg} /$ day), and clonazepam (dosage: $0.1 \mathrm{mg} / \mathrm{kg} /$ day). He was followed in PICU because of sepsis and metabolic instability. On physical examination, his vital findings were as follows: body temperature $36.5^{\circ} \mathrm{C}$, pulse $140 / \mathrm{min}$, respiration rate $40 / \mathrm{min}$ under mechanical ventilation. Bitemporal narrowing, periorbital puffiness, down-slanted palpebral fissures, sparse eyebrows, round facies, broad nasal bridge, bulbous nasal tip, prominent filtrum, thin lips, micrognathia, and generalized edema were observed (Fig. 1A). He had neither contractures nor rigidity. No neonatal reflexes were elicited, and myoclonic jerks were prominent. During the clinical course, separation from the mechanic ventilator was not successful due to insufficient spontaneous breathing. A tracheostomy was performed at the age of 2 months. He had a head circumference of $39 \mathrm{~cm}(-0.51 \mathrm{SD})$ at the age of 2 months. Gastrostomy feeding was initiated at the age of 2 months due to his inability to swallow. Initial EEG findings were consistent with left frontal focal epileptic activity (Fig. 1B). Focal epileptic activity was seen as migrating from one brain region to another. Generalized epileptic activities were also observed during the follow-ups. EEGs at the age of 2, 2.5 , and 3 months demonstrated widespread deceleration of the background. Due to süper-refractory and migrating focal seizures, video EEG monitoring was performed and antiepileptic treatment was revised to include phenobarbital (dosage: $8 \mathrm{mg} / \mathrm{kg} /$ day), clobazam (dosage: $1 \mathrm{mg} /$ $\mathrm{kg} /$ day), midazolam (dosage: $16 \mu \mathrm{cg} / \mathrm{kg} / \mathrm{m}$ ), and ketamine (dosage: $4 \mathrm{mg} / \mathrm{kg} / \mathrm{h}$ ). In his clinical course, his various 
A
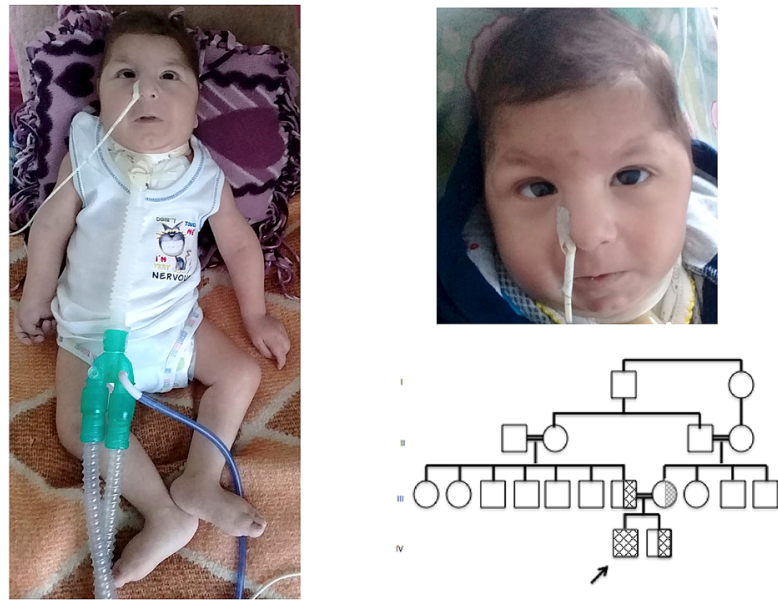

C

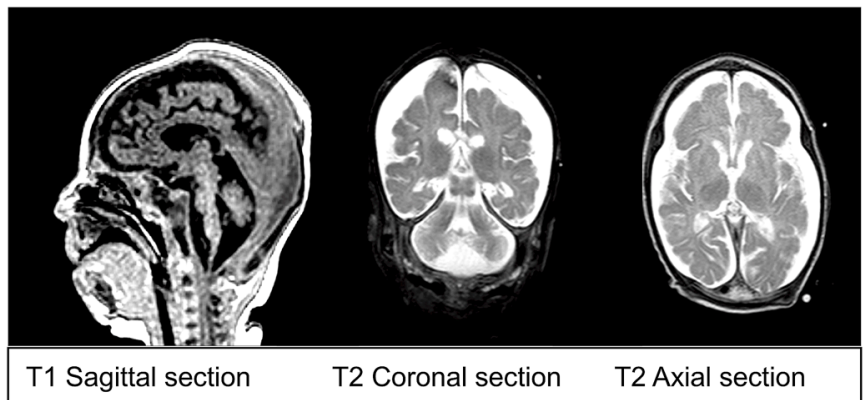

B
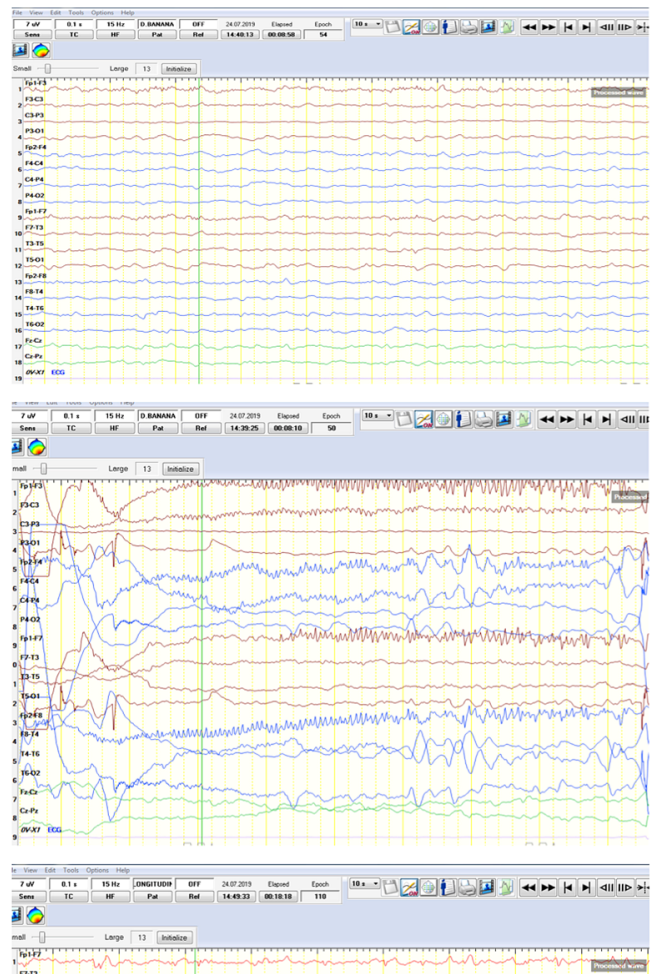

칭

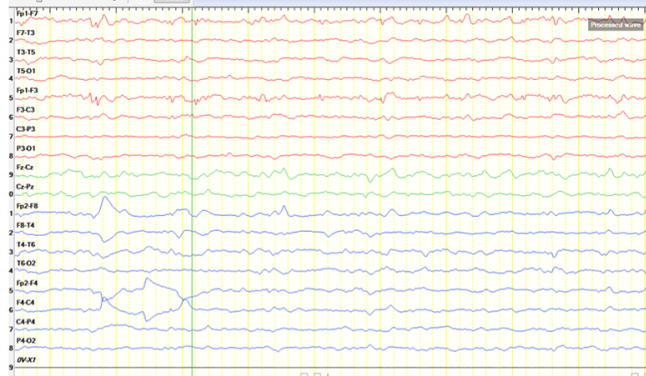

Fig. 1 Clinical photographs, pedigree, EEG, and cranial MRI of the patient. A Dysmorphic features of the affected patient; note bitemporal narrowing, periorbital puffiness, down-slanted palpebral fissures, sparse eyebrows, round facies, broad nasal bridge, bulbous nasal tip, prominent philtrum, thin lips, and micrognathia, pedigree of the patient. B EEGs of the patient: (a) ground rhythm consists of slow and disorganized amplitude theta rhythm, superposed, low-amplitude fast activity according to age. (b) during this period, a low-amplitude fast beta activity, which started from the left hemisphere frontal region and reflected on the left hemisphere electrodes, was observed for 5-6 s, followed by an asymmetric tonic ictal seizure record with right-hand rotation, and then a few beat clones in the right arm. (c) in the Fp1-Fp3 electrode position, low-amplitude thorn slow waves at a frequency of $1-2 \mathrm{~Hz}$ were observed in interictal activity. $\mathbf{C}$ Brain magnetic resonance imaging demonstrating atrophic corpus callosum, cortical laminas necrosis in both occipital and superior parietal lobes, simple spiral pattern in cortical structures, hypomyelination and brainstem, and cerebellar vermis hypoplasia compatible with pontocerebellar hypoplasia convulsions consisted of myoclonic, clonic-tonic, and variants, for which multiple antiepileptic treatments including phenobarbital and clonazepam were not effective. He had a weight of $5400 \mathrm{~g}(-2.38 \mathrm{SD})$ at the age of 5 months and his antiepileptic therapy was reedited to include carbamazepine (dosage: $20 \mathrm{mg} / \mathrm{kg} / \mathrm{day}$ ) and lacosamide (dosage: $8 \mathrm{mg} / \mathrm{kg} /$ day). Additionally, a ketogenic diet was also started. His seizures continued to be refractory with innumerable episodes per day. Laboratory examination including metabolic work-up did not reveal any abnormalities. Ophthalmological evaluation was normal. Echocardiogram revealed patent foramen ovale, minimal mitral, and aortic insufficiency. Abdomen ultrasound showed ascites. Brain magnetic resonance imaging demonstrated atrophic corpus callosum, hypomyelination, cortical laminar necrosis in both occipital and superior parietal lobes, simple spiral pattern in cortical structures, and brainstem and cerebellar vermis hypoplasia compatible with pontocerebellar hypoplasia (Fig. 1C). Symptoms rapidly progressed to intractable myoclonic seizures, bouts of apnea and bradycardia, and arrest of head growth, with no acquisition of 
developmental milestones. Despite supportive treatment, the patient died at the age of 7.5 months.

\section{Molecular results}

A novel homozygous BRATl variant, c. $1499-1 \mathrm{G}>\mathrm{T}$, located at the consensus splice acceptor site of intron 11, was identified in the DNA sample of the proband after CES analysis. In accordance with ACMG criteria, this variant was classified as "pathogenic" (PVS1, PM2, PP3). The variant was not found in publicly available databases, including the gno$\mathrm{mAD}$ database, or in our in-house control clinical exomes. In silico predictions indicating that the variant is probably pathogenic in its effect on pre-mRNA splicing were verified by genetic analysis based on reverse transcription of the patient's RNA, followed by PCR amplification and Sanger sequencing performed on cDNA. The sequencing revealed that the c.1499-1G $>\mathrm{T}$ variant disrupts the original splice acceptor site and activates a cryptic splice site only two nucleotides downstream from the variant site. This changes the first two nucleotides of exon 12 to be deleted, leading to a frameshift (Glu500Alafs*36) in the mRNA of the BRAT1 (Fig. 2). Further analysis of the parents' gDNA revealed the heterozygosity of the detected variant, which is consistent with autosomal-recessive inheritance.

\section{Discussion}

In this study, we have described a patient who presented with a severe phenotype of intractable epilepsy beginning on the first day of life, and we have demonstrated the functional effect of a novel homozygous splice acceptor site c.1499$1 \mathrm{G}>\mathrm{T}$ variant in $B R A T 1$ on the splicing machinery. Because disorders caused by $B R A T 1$ variants are manifested in a broad range of a clinical spectrum, ranging from the most severe clinic RMFSL to isolated CA, [10, 11] some authors have suggested renaming this spectrum "BRAT1-related neurodevelopmental disorders" $[8,12]$. To our knowledge, 21 publications report BRAT1-related neurodevelopmental disorders. Patients with severe clinical form are depicted and compared to the present patient in Table 1.

It has been suggested that the phenotypic spectrum of BRAT1-related disorders is associated with the type, localization, domain, and zygosity of the identified variant $[4,11$, 13]. In addition, previous reports have speculated that there could be a genotype-phenotype correlation among patients such that homozygous frameshift variants are associated with a more severe RMFSL phenotype, whereas in-frame deletions or missense variants outside of important domains in homozygous or compound heterozygous states are associated with a milder NEDCAS phenotype [11]. This report further contributes to genotype-phenotype correlation, since, as we observed in the present patient, the identified
Fig. 2 A schematic representation of the BRAT1 gene and protein. a BRAT1 protein is 821 amino acids in length, with the arrangement of the main domains: CIDE-N (N-terminal of an cell deathinducing DFF45-like effector) domain (light blue) and two HEAT (Huntingtin, Elongation factor 3 , A subunit of protein phosphatase $2 \mathrm{~A}$, and TOR1) repeat domains (blue). A schematic view was adopted from reference no: [3]. b The chromatogram and schematic representation of wild-type and mutated sequences illustrate the disrupted acceptor splice site at position -1 of intron 11 , which leads to a frameshift in the cDNA sample
A
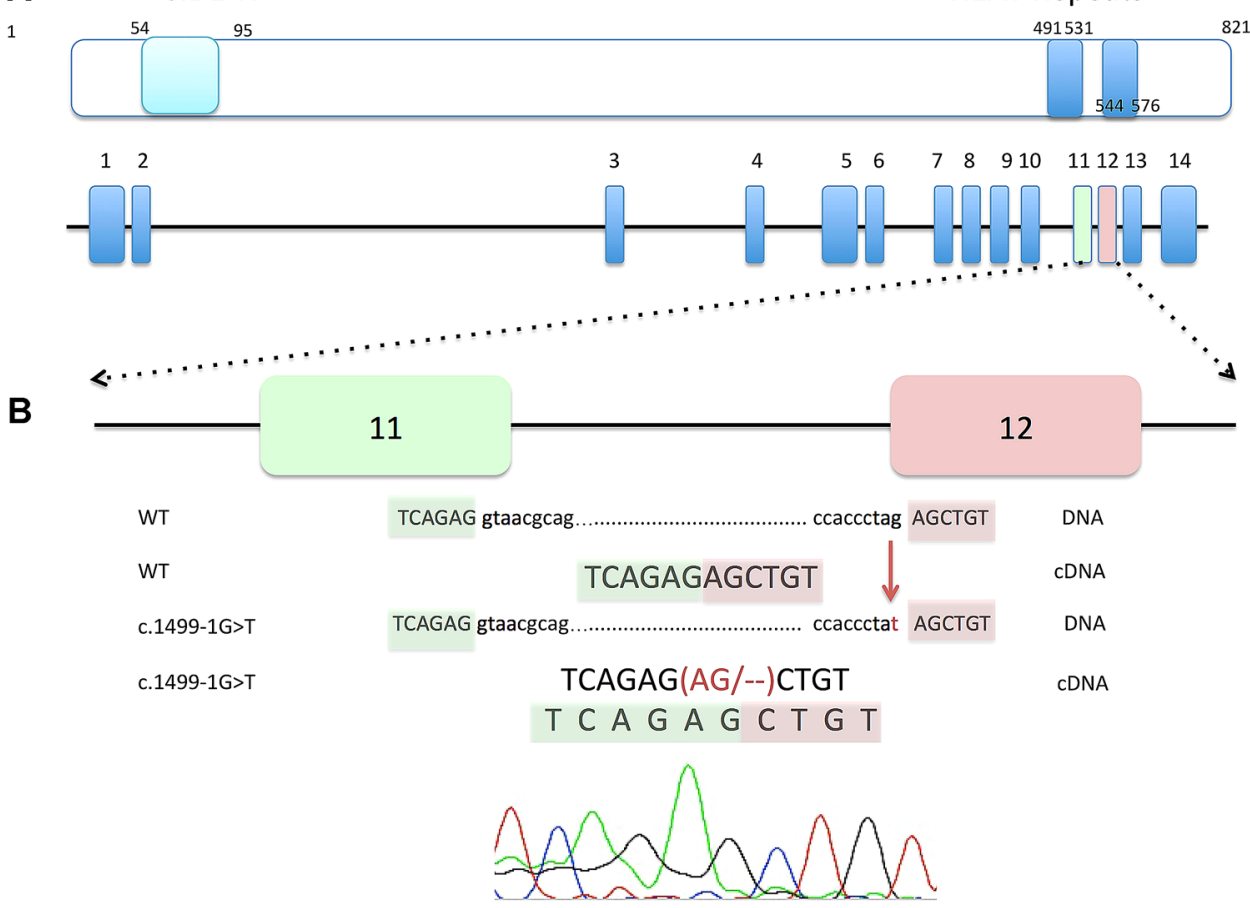


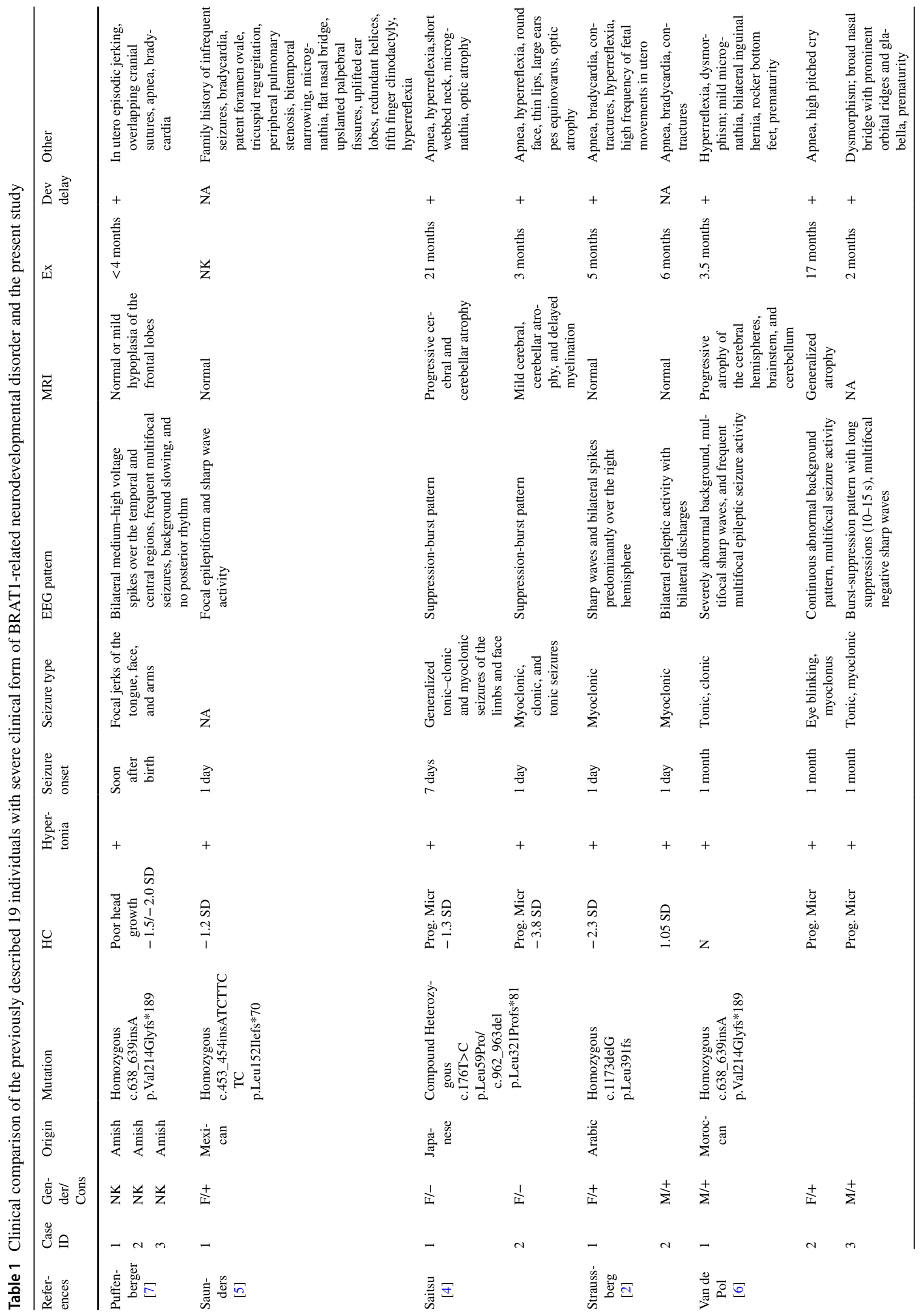




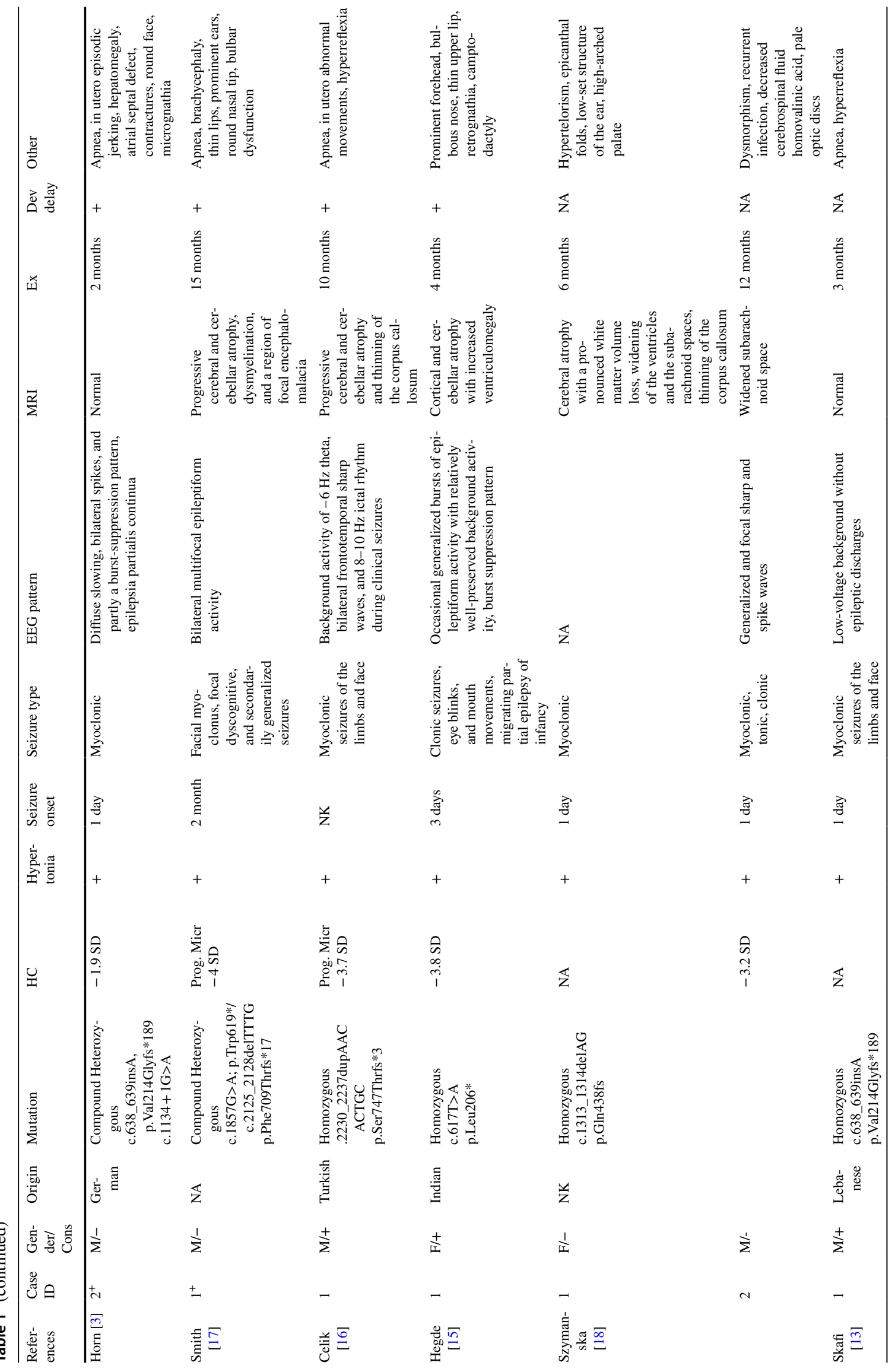




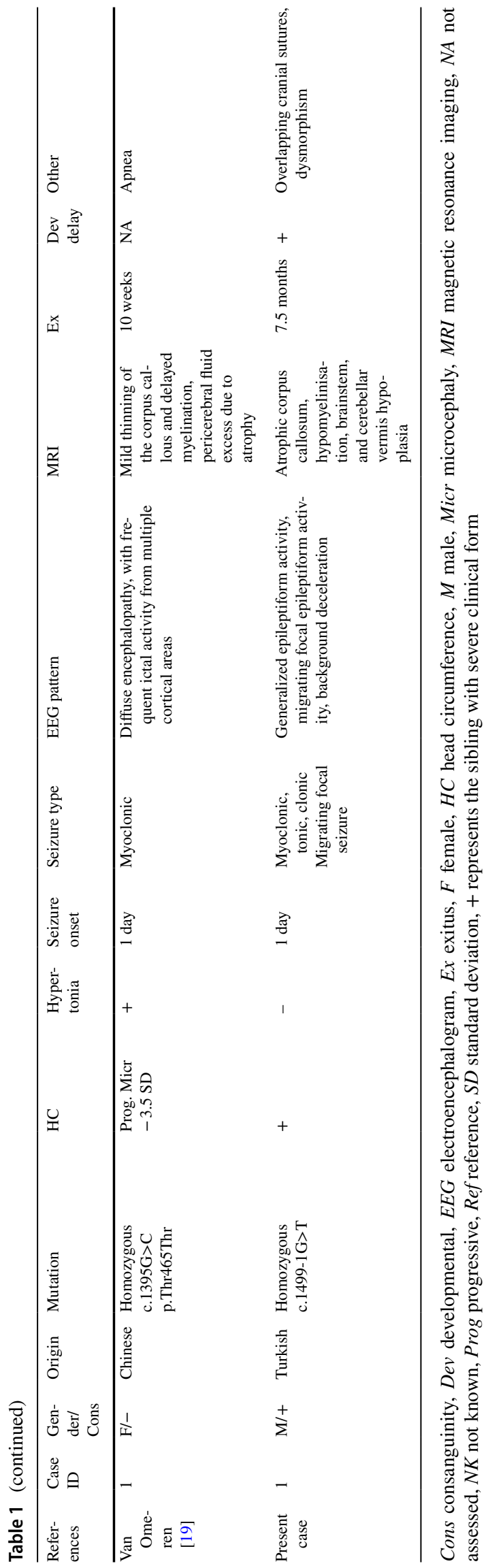

splice variant results in a loss of function of the protein and leads to a more severe form.

Variants that affect pre-mRNA splicing are one of the most common causes of hereditary disorders [14]. Although donor-site splice variants of $B R A T 1$ were previously reported in a compound heterozygous state, functional studies, including studies of cDNA sequencing, have not yet been performed $[3,12]$. In the present study, however, we did perform cDNA sequencing, even though we assumed that mRNA would undergo nonsense-mediated decay (NMD). Interestingly, cDNA sequencing of the affected patient in this study showed that the c.1499-1G $>\mathrm{T}$ variant activates a cryptic 3' splice site only two nucleotides downstream from the pathogenic variant site as the first two nucleotides of the adjacent exon are A and G, respectively. The cryptic 3' splice site was created using the first two nucleotides of the adjacent exon, resulting in the deletion of these two nucleotides and the creation of a frameshift. We thereby functionally demonstrated that the identified homozygous splice variant is a null allele and is associated with the severe phenotypic form of BRAT1-related neurodevelopmental disorders.

The phenotype of the present patient differed from the reported patients with severe form of BRAT1-related neurodevelopmental disorders, in terms of rigidity, EEG, and MRI findings. First, he exhibited all major clinical signs except rigidity. Second, unlike most patients reported in the literature, he presented with migrating epilepsy. Finally, although progressive cerebral and cerebellar atrophy were demonstrated in the previously reported patients [4, 15-19], we observed pontocerebellar hypoplasia $(\mathrm{PCH})$ in the present patient. We excluded additional variants in $28 \mathrm{PCH}$ genes (Online Resource 2) including TSEN54 that could cause clinical findings similar to RMFSL [20]. BRAT1 knockdown models result in p53-mediated apoptosis, suggesting that BRAT1 may regulate cell growth and apoptosis [7]. The pontocerebellar hypoplasia seen in the present patient may therefore be related to the increased apoptosis observed in patients with PCH3 [21].

\section{Conclusion}

This research has further expanded the mutational and phenotypic spectrum of BRAT1-related neurodevelopmental disorders by documenting a case of severe clinical presentation of migrating focal seizures and pontocerebellar hypoplasia in the absence of rigidity. Patients presenting with seizures occurring very early in life, especially on the first day of life, even without rigidity, should be suspected of harboring a pathogenic BRATl variant. 
Acknowledgements We thank the family for participating in our research. The authors would like to acknowledge the technical support by Funda Aydın and Esra Cesur.

Funding This research did not receive any specific Grant from funding agencies in the public, commercial, or not-for-profit sectors.

\section{Compliance with ethical standards}

Conflict of interest All authors declare that they have no conflict of interest.

Ethical approval All procedures performed in studies involving human participants were in accordance with the ethical standards of the institutional and/or national research committee and with the 1964 Helsinki declaration and its later amendments or comparable ethical standards.

Informed consent Informed consent was obtained from all individual participants included in the study.

\section{References}

1. McTague A, Howell KB, Cross JH, Kurian MA, Scheffer IE (2016) The genetic landscape of the epileptic encephalopathies of infancy and childhood. Lancet Neurol 15(3):304-316

2. Straussberg R, Ganelin-Cohen E, Goldberg-Stern H, Tzur S, Behar DM, Smirin-Yosef $P$ et al (2015) Lethal neonatal rigidity and multifocal seizure syndrome-report of another family with a BRAT1 mutation. Eur J Paediatr Neurol 19(2):240-242

3. Horn D, Weschke B, Knierim E, Fischer-Zirnsak B, Stenzel W, Schuelke M et al (2016) BRAT1 mutations are associated with infantile epileptic encephalopathy, mitochondrial dysfunction, and survival into childhood. Am J Med Genet A 170(9):2274-2281

4. Saitsu H, Yamashita S, Tanaka Y, Tsurusaki Y, Nakashima M, Miyake N et al (2014) Compound heterozygous BRAT1 mutations cause familial Ohtahara syndrome with hypertonia and microcephaly. J Hum Genet 59(12):687-690

5. Saunders CJ, Miller NA, Soden SE, Dinwiddie DL, Noll A, Alnadi NA et al (2012) Rapid whole-genome sequencing for genetic disease diagnosis in neonatal intensive care units. Sci Transl Med 4(154): 154 ra135

6. van de Pol LA, Wolf NI, van Weissenbruch MM, Stam CJ, Weiss JM, Waisfisz Q et al (2015) Early-onset severe encephalopathy with epilepsy: the BRAT1 gene should be added to the list of causes. Neuropediatrics 46(06):392-400

7. Puffenberger EG, Jinks RN, Sougnez C, Cibulskis K, Willert RA, Achilly NP et al (2012) Genetic mapping and exome sequencing identify variants associated with five novel diseases. PLoS ONE 7(1):e28936
8. Hanes I, Kozenko M, Callen DJ (2015) Lethal neonatal rigidity and multifocal seizure syndrome - a misnamed disorder? Pediatr Neurol 53(6):535-540

9. Mundy SA, Krock BL, Mao R, Shen JJ (2016) BRAT1-related disease-identification of a patient without early lethality. Am J Med Genet A 170(3):699-702

10. Scheffer IE, Boysen KE, Schneider AL, Myers CT, Mehaffey MG, Rochtus AM et al (2019) BRAT 1 encephalopathy: a recessive cause of epilepsy of infancy with migrating focal seizures. Dev Med Child Neurol 62:1096-1099

11. Valence S, Cochet E, Rougeot C, Garel C, Chantot-Bastaraud S, Lainey E et al (2019) Exome sequencing in congenital ataxia identifies two new candidate genes and highlights a pathophysiological link between some congenital ataxias and early infantile epileptic encephalopathies. Genet Med 21(3):553

12. Srivastava S, Olson HE, Cohen JS, Gubbels CS, Lincoln S, Davis BT et al (2016) BRAT1 mutations present with a spectrum of clinical severity. Am J Med Genet A 170(9):2265-2273

13. Skafi O, Fawaz A, Merhi B, Jouni H, Mansour S (2018) Rigidity with multifocal seizure syndrome, lethal neonatal in a Lebanese neonate. A rare case report. J Pediatr Disord Neonatal Care 1:106

14. Anna A, Monika G (2018) Splicing mutations in human genetic disorders: examples, detection, and confirmation. J Appl Genet 59(3):253-268

15. Hegde AU, Sanghvi KP, Karnavat PK, Jalan AB (2017) BRCA1associated ataxia telangiectasia mutated activation-1 mutation: an addition to the early infantile epileptic encephalopathy panel. J Clin Neonatol 6(3):200

16. Celik Y, Okuyaz C, Arslankoylu AE, Ceylaner S (2017) Lethal neonatal rigidity and multifocal seizure syndrome with a new mutation in BRAT1. Epilepsy Behav Case Rep 8:31-32

17. Smith NJ, Lipsett J, Dibbens LM, Heron SE (2016) BRAT1 associated neurodegeneration: intra-familial phenotypic differences in siblings. Am J Med Genet A 170(11):3033-3038

18. Szymańska K, Laure-Kamionowska M, Szczałuba K, Koppolu A, Furmanek M, Kuśmierska K et al (2018) Clinico-pathological correlation in case of BRAT1 mutation. Folia Neuropathol 56(4):362-371

19. Van Ommeren RH, Gao AF, Blaser SI, Chitayat DA, Hazrati L-N (2018) BRAT1 mutation: the first reported case of Chinese origin and review of the literature. J Neuropathol Exp Neurol 77(12):1071-1078

20. Grillo E (2016) BRAT1 and TSEN54: important roles in basic cellular processes and similar phenotypes. Pediatr Neurol 57:e1

21. van Dijk T, Baas F, Barth PG (2018) What's new in pontocerebellar hypoplasia? An update on genes and subtypes. Orphanet J Rare Dis 13(1):92

Publisher's Note Springer Nature remains neutral with regard to jurisdictional claims in published maps and institutional affiliations. 